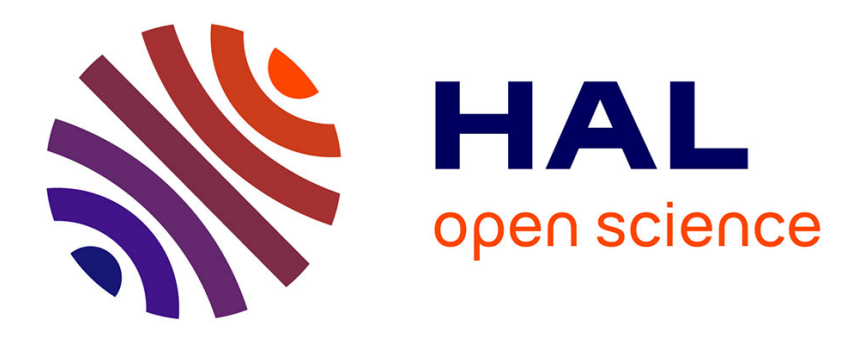

\title{
Optical determination of viscoelastic properties of nematic OHMBBA
}

\author{
J.P. van Der Meulen, R.J.J. Zijlstra
}

\section{To cite this version:}

J.P. van Der Meulen, R.J.J. Zijlstra. Optical determination of viscoelastic properties of nematic OHMBBA. Journal de Physique, 1982, 43 (2), pp.411-420. 10.1051/jphys:01982004302041100 . jpa00209409

\section{HAL Id: jpa-00209409 https://hal.science/jpa-00209409}

Submitted on 1 Jan 1982

HAL is a multi-disciplinary open access archive for the deposit and dissemination of scientific research documents, whether they are published or not. The documents may come from teaching and research institutions in France or abroad, or from public or private research centers.
L'archive ouverte pluridisciplinaire HAL, est destinée au dépôt et à la diffusion de documents scientifiques de niveau recherche, publiés ou non, émanant des établissements d'enseignement et de recherche français ou étrangers, des laboratoires publics ou privés. 
Classification

Physics Abstracts

$61.30 \mathrm{E}$

\title{
Optical determination of viscoelastic properties of nematic OHMBBA
}

\author{
J. P. van der Meulen and R. J. J. Zijlstra \\ Physics Laboratory, Fluctuations Phenomena Group, \\ University of Utrecht, Princetonplein 5, 3584 CC Utrecht, The Netherlands
}

(Reçu le 4 mai 1981, révisé le 29 septembre, accepté le 9 octobre 1981)

\begin{abstract}
Résumé. - Nous avons mesuré des paramètres viscoélastiques du nématique OHMBBA en fonction de la température. Nos expériences se basent sur la technique homodyne avec laquelle on peut détecter la densité spectrale de la lumière laser diffusée par des cristaux liquides. On peut décrire le coefficient de la viscosité de torsion en fonction de la température, du paramètre d'ordre et d'une énergie d'activation. Nous obtenons le rapport entre les constantes élastiques de divergence et de torsion par deux méthodes : 1) en déterminant la section efficace de diffusion en fonction du vecteur d'onde de diffusion et 2) en faisant une analyse de la densité spectrale dans le mode mélangé (torsion plus divergence). Les résultats obtenus avec ces deux méthodes concordent, mais ils diffèrent des résultats obtenus avec la technique de Freedericksz. Nous discutons ce désaccord brièvement.
\end{abstract}

\begin{abstract}
Experimental values are reported for the splay/twist viscoelastic ratios of OHMBBA in the nematic region as a function of temperature. In the experiments the homodyne self-beat technique was used to detect noise intensity spectra of laser light scattered by the nematic liquid crystal. The measured rotational viscosity can be described as a function of the temperature, the order parameter and an activation energy. The splay/twist ratio of the elastic constants was determined by measuring the scattered cross section as a function of the scattering wave vector and by making a noise analysis in the mixture region. The results obtained with each method are in agreement, but differ from Freedericksz transition data. This discrepancy is discussed.
\end{abstract}

1. Introduction. - Light scattered by local director fluctuations of an aligned nematic liquid crystal (NLC) yields information about the viscoelastic properties of that crystal $[1,2,3]$. In this paper we describe light scattering experiments on OHMBBA, which produce data about the splay/twist elastic ratio and the splay and the twist viscoelastic ratios.

To our knowledge no viscosity data have been published so far. However, we were able to compare values for the elastic constants with those obtained from measurements of Freedericksz transitions [4].

OHMBBA (o-hydroxy-p-methoxybenzylidene- $\mathrm{p}^{\prime}$ butylaniline) has the following molecular structure :

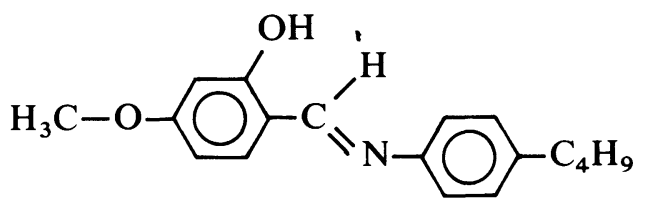

Its nematic range is from $T_{\mathrm{SN}}=44{ }^{\circ} \mathrm{C}$ to $T_{\mathrm{NI}}=64.6^{\circ} \mathrm{C}$ at $1 \mathrm{~atm}$.

The splay/twist viscoelastic ratios were obtained from the line broadening of the scattered laser light, caused by orientational fluctuations of the director [5].
These ratios were determined as a function of temperature in the entire nematic range and at $1 \mathrm{~atm}$. pressure. In addition it seemed worthwhile to study the dependence of these ratios on orientational order, since the literature data on this subject are ambiguous $[6,7]$. From our measurements we çould also determine the total scattering cross section, from which in turn we found the splay/twist curvature elastic constants. These ratios were also determined from noise measurements done in the mixture region, where a mixture of splay and twist distortion fluctuations can be observed. In this region the splay/twist mixture component dominates over the pure splay and pure twist component. In the so-called mixture mode the splay/twist mixture component is at its maximum at a specific scattering angle, $\theta_{\text {mix }}$, which can be determined. Since $\theta_{\text {mix }}$ is a function of well-known optical parameters and the splay/twist elastic ratio, the latter can be determined by measuring $\theta_{\text {mix }}$. In the theory of light scattering in nematics it is tacitly assumed that the sample dimensions are very large with respect to the wavelength of light. In this paper, however, the dimensions of the scattering volume are taken to be finite. 
2. Experimental arrangement for the investigation of scattered light. - The experimental arrangement, shown in figure 1, is a normal homodyne self-beat set-up, which is strictly the same as that used by van Eck et al. [8]. See their paper for a detailed description. The light source is a $5 \mathrm{~mW}$ He-Ne laser $\left(\lambda_{0}=632.8 \mathrm{~nm}\right.$, Spectra Physics model 120). The unfocussed light beam has a cross section of $1 \mathrm{~mm}^{2}$. The scattered light is detected by a photomultiplier tube (PMT) placed about $0.50 \mathrm{~m}$ from the scattering centre. The set-up is placed in a darkened room, and with the help of a set of diaphragms, the detection of stray-light is avoided. Since the diaphragm in front of the PMT has a diameter of $4 \mathrm{~mm}$, the solid angle of observation has a constant value of about $5 \times 10^{-5}$ sterad. The fluctuating anode current of the PMT is fed into a real-time frequency analyser (Brüel and Kjaer, type 3347), which displays the noise spectrum in the frequency range $12.5 \mathrm{~Hz}$ to $63 \mathrm{kHz}$.

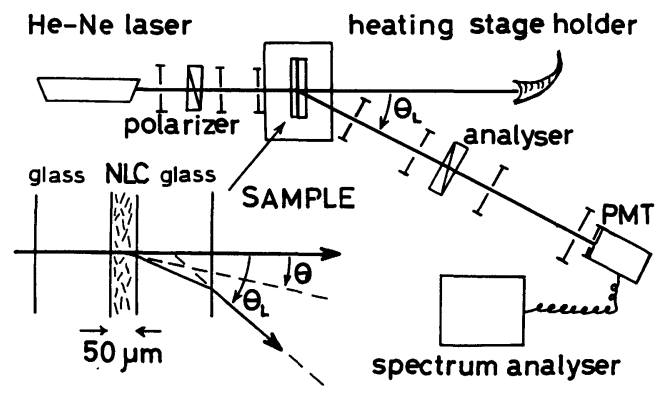

Fig. 1. - Experimental set-up.

The sample we used contained OHMBBA which was planar orientated simply by rubbing the glass walls of the sample. The space between the glass walls was $50 \mu \mathrm{m}$ and was maintained by tungsten ribbon and a special epoxy resin (Lekutherm E 320). The sample was placed between heating stages (Mettler PF 52), which kept the temperature constant within $0.01{ }^{\circ} \mathrm{C}$. The sample temperature was not influenced by absorption of laser light. This was ascertained by varying the intensity of the light source and by measuring the influence of the light scattering exactly in the nematicisotropic transition region. No change in the scattering pattern was observed.

The laboratory scattering angle, $\theta_{\mathrm{L}}$, i.e. the angle outside the scattering medium, could be varied between $0^{\circ}$ and $30^{\circ}$. The polarization vectors $i$ and $f$ of the incoming and detected scattered light respectively could be chosen at will by means of a polarizer and analyser. Appropriate choices for $\mathbf{i}$ and $\mathbf{f}$ with respect to the orientation of the NLC made it possible for us to measure pure splay and pure twist effects [8]. The configuration we used is shown in figure 2 .

3. Theory. - 3.1 Light SCATTERING BY NLC. The optical spectrum of the scattered light is the

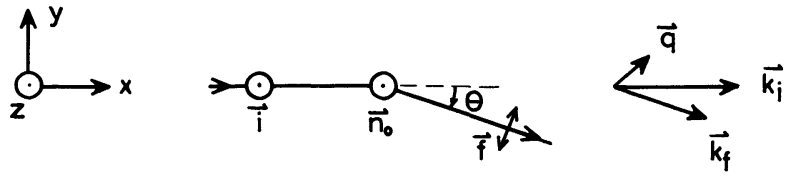

Fig. 2. - Scattering diagram of the experimental configuration for splay/twist-mode detection.

Fourier cosine transform of the auto-correlation function of the detected electric field [2]. This autocorrelation function in turn is proportional to the following auto-correlation function

$$
A(\mathbf{q}, t)=\left\langle\delta \varepsilon_{i f}^{*}(\mathbf{q}, 0) \delta \varepsilon_{i f}(\mathbf{q}, t)\right\rangle,
$$

where

$$
\delta \varepsilon_{i f}(\mathbf{q}, t)=\mathbf{f} . \delta \varepsilon(\mathbf{q}, t) . \mathbf{i},
$$

with $\mathbf{i}$ and $\mathbf{f}$ the unit vectors marking the directions of polarization of the incoming and scattered laser light respectively. The vector $\mathbf{q}$ is the scattering wave vector, i.e. $\mathbf{q}=\mathbf{k}_{i}-\mathbf{k}_{f}$ (see Fig. 2). The tensor $\delta \varepsilon(\mathbf{q}, t)$ is given by

$$
\delta \varepsilon(\mathbf{q}, t)=\frac{1}{V} \int_{V} \mathrm{~d} \mathbf{r} \mathrm{e}^{i \mathbf{q} . \mathbf{r}} \delta \varepsilon(\mathbf{r}, t),
$$

where $\delta \varepsilon(\mathbf{r}, t)$ is the local fluctuation of the dielectric tensor with respect to the average thermal equilibrium value $\varepsilon_{0}$ and $V$ is the scattering volume.

The mean orientation of the rod-like molecules, called the director, is given by the unit vector $\mathbf{n}_{0}$. The frame of reference we have chosen is shown in figure 2; for our configuration we defined the director of the NLC as :

$$
\mathbf{n}_{0}=\left(0,0, n_{0}\right),
$$

where average values are denoted by the subscript 0 . Assuming that fluctuations around $\mathbf{n}_{0}$ are small, we have in first order :

$$
\delta n_{z}(\mathbf{r}, t)=0
$$

and for $i=x, y$ :

$$
\mathbf{n}_{i}(\mathbf{r}, t)=\delta \mathbf{n}_{i}(\mathbf{r}, t)
$$

For our configuration we now have

$$
\delta \varepsilon(\mathbf{q}, t)=\Delta \varepsilon\left(\begin{array}{lll}
0 & 0 & \delta n_{x}(\mathbf{q}, t) \\
0 & 0 & \delta n_{y}(\mathbf{q}, t) \\
\delta n_{x}(\mathbf{q}, t) & \delta n_{y}(\mathbf{q}, t) & 0
\end{array}\right),
$$

where

$$
\delta \mathbf{n}(\mathbf{q}, t)=\frac{1}{V} \int_{V} \mathrm{dr} \mathrm{e}^{i \mathbf{q} . \mathbf{r}} \delta \mathbf{n}(\mathbf{r}, t),
$$

and $\Delta \varepsilon=\varepsilon_{\|}-\varepsilon_{\perp} ; \varepsilon_{\|}$and $\varepsilon_{\perp}$ are the dielectric constants for electric fields directed parallel and perpen- 
dicular to $\mathbf{n}_{0}$ respectively. Hence we get for equation (1) :

$$
\begin{array}{r}
A(\mathbf{q}, t)=(\Delta \varepsilon)^{2}\left\{G_{x}^{2}\left\langle\delta n_{x}(\mathbf{q}, t) \delta n_{x}^{*}(\mathbf{q}, 0)\right\rangle+\right. \\
\left.G_{y}^{2}\left\langle\delta n_{y}(\mathbf{q}, t) \delta n_{y}^{*}(\mathbf{q}, 0)\right\rangle\right\},
\end{array}
$$

where

$$
G_{\alpha} \equiv f_{\alpha} i_{z}+i_{\alpha} f_{z} \quad(\alpha=x, y) .
$$

The quantity $G_{\alpha}$ is called an optical geometric factor. Thus the optical spectrum is directly related to the auto-correlation function of $\mathbf{n}(\mathbf{q}, t)$.

\subsubsection{Finite dimensions of the scattering volume. -} Because in our experiment the position of the director was fixed at the glass walls we can express the fluctuations of the local director as follows :

$$
\delta \mathbf{n}(\mathbf{r}, t)=\frac{1}{2} \sum_{m}\left(\delta \mathbf{n}_{m}(t)\right)_{0} \mathrm{e}^{i \mathbf{k}_{m} \cdot \mathbf{r}},
$$

with

$$
\left(k_{i}\right)_{m}=m \frac{\pi}{d_{i}} \quad(i=x, y, z)
$$

These relations hold because of the boundary conditions. The term $d_{i}$ is the size of the sample in the $i=x, y$ or $z$ direction. In these light scattering experiments the scattering wave vector $\mathbf{q}$ is related to the wave vector $\mathbf{k}_{m}$, as will be shown.

Although $\mathbf{k}_{m}$ has discrete values, the intensity of the scattered light is a continuous function of the scattering angle; this is because the dimensions of the scattering volume are finite. Therefore we shall extend the light scattering analysis by taking the finite dimensions of the sample into account. When we substitute equation (7) into (4) we get :

$$
\delta \mathbf{n}(\mathbf{q}, t)=\frac{1}{V} \frac{1}{2} \sum_{m}\left(\delta \mathbf{n}_{m}(t)\right)_{0} \int_{V} \mathrm{~d} \mathbf{r} \mathrm{e}^{i\left(\mathbf{q}-\mathbf{k}_{m}\right) \cdot \mathbf{r}} .
$$

The integral can be written as a product of three integrals :

$$
\begin{aligned}
\frac{1}{V} \int_{V} \mathrm{~d} \mathbf{r} \mathrm{e}^{i\left(\mathbf{q}-\mathbf{k}_{m}\right) \cdot \mathbf{r}}=\frac{1}{x_{0}} \int_{-\frac{1}{2} x_{0}}^{\frac{1}{2} x_{0}} \mathrm{~d} x \mathrm{e}^{i\left(q_{x}-\left(k_{x}\right)_{m}\right) \cdot x} \times \\
\times \frac{1}{y_{0}} \int_{-\frac{1}{2} y_{0}}^{\frac{1}{2} y_{0}} \mathrm{~d} y \mathrm{e}^{i\left(q_{y}-\left(k_{y}\right)_{m}\right) \cdot y} \\
\times \frac{1}{z_{0}} \int_{-\frac{1}{2} z_{0}}^{\frac{1}{2} z_{0}} \mathrm{~d} z \mathrm{e}^{i\left(q_{z}-\left(k_{z}\right)_{m}\right) \cdot z},
\end{aligned}
$$

where $x_{0}, y_{0}$ and $z_{0}$ are the sizes of the scattering volume. For our sample, where $y_{0} \approx z_{0} \approx 1 \mathrm{~mm}$ it holds that $y_{0}, z_{0} \gg \lambda_{0}$. Hence we can write

$$
\begin{array}{r}
\frac{1}{y_{0}} \int_{-\frac{1}{2} y_{0}}^{\frac{1}{2} y_{0}} \mathrm{~d} y \mathrm{e}^{i\left(q_{y}-\left(k_{y}\right)_{m}\right) \cdot y} \times \frac{1}{z_{0}} \int_{-\frac{1}{2} z_{0}}^{\frac{1}{2} z_{0}} \mathrm{~d} z \mathrm{e}^{i\left(q_{z}-\left(k_{z}\right)_{m}\right) \cdot z}= \\
=\delta_{q_{y},\left(k_{y}\right)_{m} \cdot \delta_{q_{z},\left(k_{z}\right)_{m}},}
\end{array}
$$

where $\delta_{i, j}$ denotes the Kronecker symbol. The thickness of the scattering volume $x_{0}=d_{x}=50 \mu \mathrm{m}$ is nevertheless large compared to $\lambda_{0}$, but too small for the $x$-th component of the integral to be considered as a delta function. However, because $d_{y}=1 \mathrm{~cm}$ and $d_{z}=3 \mathrm{~cm}$ the spacings of $\left(k_{y}\right)_{m}$ and $\left(k_{z}\right)_{m}$ are too small to be resolved by our experimental set-up. Unlike $\left(k_{x}\right)_{m}$, which can only have discrete values, as is shown in figure $3,\left(k_{y}\right)_{m}$ and $\left(k_{z}\right)_{m}$ were taken as varying continuously.

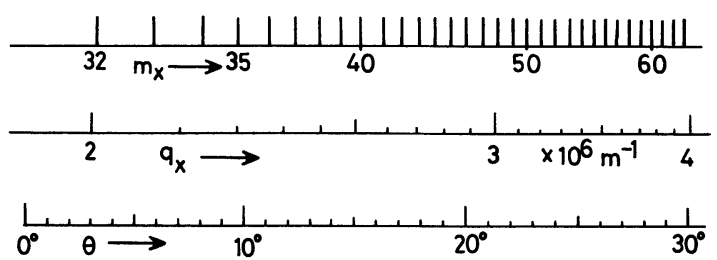

Fig. 3. - Example of a plot for comparing $\left(k_{x}\right)_{m}, q_{x}$ and the scattering angle $\theta$ for $n_{\perp}=1.5$ and $n_{\|}=1.7$.

Hence,

$$
\begin{array}{r}
\frac{1}{V} \cdot \int_{V} \mathrm{dr} \mathrm{e}^{i\left(\mathbf{q}-\mathbf{k}_{m}\right) \cdot \mathbf{r}}=\left\{\frac{\sin \left(\frac{1}{2} x_{0}\left(q_{x}-\left(k_{x}\right)_{m}\right)\right)}{\frac{1}{2} x_{0}\left(q_{x}-\left(k_{x}\right)_{m}\right)}\right\} \times \\
\times \delta_{q_{y},\left(k_{y}\right)_{m}} \cdot \delta_{q_{z},\left(k_{z}\right)_{m}} .
\end{array}
$$

Using this result equation (9) becomes :

$$
\delta \mathbf{n}(\mathbf{q}, t)=\sum_{m} \delta \mathbf{n}_{\left(k_{x}\right)_{m}, q_{y}, q_{z}}(t)\left\{\frac{\sin \left(\frac{1}{2} x_{0}\left(q_{x}-\left(k_{x}\right)_{m}\right)\right)}{\frac{1}{2} x_{0}\left(q_{x}-\left(k_{x}\right)_{m}\right)}\right\}
$$

This means that $\delta \mathbf{n}(\mathbf{q}, t)$ is the summation over all $\delta \mathbf{n}\left(\left(k_{x}\right)_{m}, q_{y}, q_{z} ; t\right)$ weighted by the function

$$
\left\{\frac{\sin \left(\frac{1}{2} x_{0}\left(q_{x}-\left(k_{x}\right)_{m}\right)\right)}{\frac{1}{2} x_{0}\left(q_{x}-\left(k_{x}\right)_{m}\right)}\right\}
$$

which has a maximum at $\left(k_{x}\right)_{m}=q_{x}$. The viscoelastic data can be determined from the relaxation times of the NLC, which in turn follow from the auto-correlation function $\left\langle\mathbf{n}_{\mathbf{k}_{m}}(t) \mathbf{n}_{\mathbf{k}_{m}}(0)\right\rangle$, as will be demonstrated later. In our light experiments, however, we measure quantities related to $\langle\mathbf{n}(\mathbf{q}, t) \mathbf{n}(\mathbf{q}, 0)\rangle$. Equation (10) provides a link between the measured quantities and the calculated ones.

If the finite dimensions of the scattering volume are taken into account in the case of cross-section measurements we obtain values which differ by $2-3 \%$ from those found when we neglected the finite dimensions, i.e.

$$
\delta \mathbf{n}(\mathbf{q}, t)=\delta \mathbf{n}_{\mathbf{k}_{m}}(t) .
$$

3.1.2 Relation between the fluctuations of the director and the scattering cross section. - The kinetic 
properties of the director are characterized by the viscoelastic ratios of the NLC. Information about these properties can be obtained from the spectral intensity of the fluctuations in the scattered light intensity. If one takes as an orthonormal base $\mathbf{e}_{1}, \mathbf{e}_{2}$, $\mathbf{e}_{3}$, where

$$
\mathbf{e}_{3} \equiv \mathbf{n}_{0}, \mathbf{e}_{2} \perp\left(\mathbf{k}_{m}, \mathbf{n}_{0}\right) \text { and } \mathbf{e}_{1} \perp\left(\mathbf{e}_{2}, \mathbf{e}_{3}\right),
$$

then the expression for the free energy becomes quadratic in $\delta \mathbf{n}_{\mathbf{k}_{m}}$ [9]. Therefore the equipartition theorem holds for every degree of freedom. In our case, according to the configuration of figure 2 where $q_{z}=0$ because $\mathbf{q} \perp \mathbf{n}_{0}$, the variance becomes [1] :

$$
\left\langle\left|\left(\delta n_{\left(k_{x}, k_{y}, 0\right)_{m}}\right)_{\alpha}\right|^{2}\right\rangle=\frac{k_{\mathrm{B}} T}{V^{\prime}\left(K_{\alpha} k_{m}^{2}\right)},
$$

where

$$
k_{m}^{2}=\left(k_{x}\right)_{m}^{2}+\left(k_{y}\right)_{m}^{2}=\left(k_{1}\right)_{m}^{2}+\left(k_{2}\right)_{m}^{2}
$$

and where $\alpha=1,2$ according to the orthonormal base $\left(e_{1}, e_{2}, e_{3}\right) . K_{1}$ and $K_{2}$ correspond to the Frank elastic constants associated with splay and twist respectively. The term $k_{\mathrm{B}}$ is the Boltzmann constant, $T$ the temperature and $V^{\prime}$ the sample volume.

The differential scattering cross section per solid angle can be written in terms of the auto-correlation function $A$ as follows [10] :

$$
\frac{\mathrm{d} \sigma}{\mathrm{d} \Omega} \sim A(\mathbf{q}, 0) .
$$

Hence if we put the results of (10) and (11) into (5) we have :

$$
\begin{aligned}
\frac{\mathrm{d} \sigma}{\mathrm{d} \Omega} \sim \sum_{m} \sum_{\alpha} \mid\left\langle\left|\left(\delta n_{\left(k_{x}\right)_{m}, q_{y}, 0}\right)_{x}\right|^{2}\right\rangle \times \\
\quad \times\left\{\frac{\sin ^{2}\left(\frac{1}{2} x_{0}\left(q_{x}-\left(k_{x}\right)_{m}\right)\right)}{\frac{1}{2} x_{0}\left(q_{x}-\left(k_{x}\right)_{m}\right)}\right\} \times G_{\alpha}^{2} \mid,
\end{aligned}
$$

where the indices $\alpha=1,2$ refer to the base $\left(\mathbf{e}_{1}, \mathbf{e}_{2}, \mathbf{e}_{3}\right)$. Note that this base depends on $\mathbf{k}_{m}$. As a consequence the summation symbols in equation (13) cannot be exchanged. In order to simplify the mathematics, however, we define only one base $\left(\mathbf{e}_{1}, \mathbf{e}_{2}, \mathbf{e}_{3}\right)$ related to $\mathbf{q}$ instead of to $\mathbf{k}_{m}$ and then the summation symbols can be exchanged. Now we can split (13) into two parts, each with its own summation. The splitting procedure will introduce an error into the calculations of $K_{\alpha}$ because of (11). In our case the estimated error in $K_{\alpha}$ is much smaller than $1 \%$. So in our experiments we can neglect this error and we can base $\left(\mathbf{e}_{1}, \mathbf{e}_{2}, \mathbf{e}_{3}\right)$ on $\mathbf{q}$ and $\mathbf{n}_{0}$. We shall give more details about the calculations of $K_{\alpha}$ in section 3.1.3.

3.1.3 Determination of the splay/twist elastic ratio by differential cross section measurements. - In section 3.1 .2 we have given the relation between the scattering cross section and the elastic constants. It follows from (11) that

$$
\left\langle\left|\left(\delta n_{\left(k_{x}\right)_{m}, q_{y}, 0}\right)_{x}\right|^{2}\right\rangle=\frac{k_{\mathrm{B}} T}{V^{\prime}} \frac{1}{K_{\alpha}\left(\left(k_{x}\right)_{m}^{2}+q_{y}^{2}\right)} .
$$

Because the mean intensity $I(q)$ of light scattered at the angle $\theta$ is proportional to $\mathrm{d} \sigma / \mathrm{d} \Omega$ we may write for (13) :

$$
I(\mathbf{q}) \sim Q(\mathbf{q}) \cdot\left(\sum_{x=1,2} \frac{G_{x}^{2}}{K_{x}}\right),
$$

where

$$
\begin{aligned}
& Q(\mathbf{q}) \equiv \sum_{m}\left\{\frac{1}{\left(\left(k_{x}\right)_{m}^{2}+q_{y}^{2}\right)}\right\} \times \\
& \times\left\{\frac{\sin \left(\frac{1}{2} x_{0}\left(q_{y}-\left(k_{x}\right)_{m}\right)\right)}{\frac{1}{2} x_{0}\left(q_{y}-\left(k_{x}\right)_{m}\right)}\right\}^{2} .
\end{aligned}
$$

The factor $Q(\mathbf{q})$ can be calculated numerically for each $\mathbf{q}$. By varying $\theta$ the well-known geometric factor $G_{\alpha}^{2}$ changes and measurements of $I(q)$ allow us to determine the ratio $K_{1} / K_{2}$.

Note that it is sufficient to measure only the relative change of $I$ as a function of the scattering angle. Hence the absolute value of $I$ is not needed.

3.1.4 Determination of the viscoelastic properties from spectral noise analysis. - The expression for the noise intensity spectrum of the scattered light obtained using our self-beat technique is given by the convolution integral of the spectrum of the scattered electric field; this expression holds if the field fluctuations are Gaussian [2]. The spectrum of the scattered electric field consists of two Lorentzians, i.e. one due to the splay deformation and the other to the twist deformation. In our configuration (see Fig. 2) we shall not measure the bend deformation, but only the splay and twist deformation [2]. Therefore, for the noise intensity spectrum of the scattered light we get

$$
\begin{aligned}
& S_{\Delta I}(\omega, \boldsymbol{q})=G_{1}^{4} \frac{\left(2 \Delta \omega_{1}\right)}{K_{1}^{2} q^{2}\left(\omega^{2}+\left(2 \Delta \omega_{1}\right)^{2}\right)}+ \\
& \text { 《splay 》 } \\
& +2 G_{1}^{2} G_{2}^{2} \frac{\left(\Delta \omega_{1}+\Delta \omega_{2}\right)}{K_{1} K_{2} q^{2}\left(\omega^{2}+\left(\Delta \omega_{1}+\Delta \omega_{2}\right)^{2}\right)} \\
& \text { 《splay/twist mixture 》 } \\
& +G_{2}^{4} \frac{\left(2 \Delta \omega_{2}\right)}{K_{2}^{2} q^{2}\left(\omega^{2}+\left(2 \Delta \omega_{2}\right)^{2}\right)} . \\
& \text { 《 twist }\rangle
\end{aligned}
$$

This spectrum consists of three Lorentzians, weighted by functions of $G_{\alpha}^{2} / K_{\alpha} q(\alpha=1,2)$. Fortunately $G_{1}$ tends to zero for small scattering angles $\left(\theta \leqq 5^{\circ}\right)$ and only the twist term remains, while for angles $\theta>20^{\circ}$, $G_{2}$ can be neglected and the splay term dominates. So in each of these two cases we have only one Lorentzian with a half-bandwidth $\Delta \omega_{1 / 2}=2 \Delta \omega_{\alpha}(\alpha=1$, 
splay; $\alpha=2$, twist), where according to the slow mode expression $u_{\mathrm{s}_{\alpha}}$ from [1] :

$$
\Delta \omega_{\alpha} \equiv u_{\mathrm{s}_{\alpha}}=\frac{K_{\alpha} q^{2}}{\eta_{\alpha}}
$$

The spectrum also consists of a fast mode term [1]. This fast mode is associated with a diffusion of vorticity. The half-bandwidth $u_{\mathrm{s}_{\mathrm{F}}}$, however, is of the order of $10^{9} \mathrm{~s}^{-1}$ and the contribution of the fast mode is much smaller than that of the slow mode, so we can neglect this term.

From equation (17) it follows that if we plot $\frac{1}{2}\left(\Delta \omega_{1 / 2}\right)_{\text {exp }}$ versus $q^{2}$ we expect straight lines with a gradient equal to the viscoelastic ratios. The scattering wave vector can be varied by varying the scattering angle $\theta$ (see Fig. 2).

In fact equation (16) should contain summation over $\mathbf{k}_{m}$, as was pointed out in section 3.1.2, but in our case the error introduced by using equation (16) is small with respect to the experimental error of about $5 \%$.

3.1.5 Determination of the splay/twist elastic ratio from the mixture mode. - Another way of deriving $K_{1} / K_{2}$ is to determine the scattering vector $q$ where the mixture contribution is at its maximum. In this case the contribution of the splay and the twist terms are equal and the bandwidth of the mixture mode is the mean of the splay and twist mode bandwidths, i.e.

$$
\left(\Delta \omega_{1 / 2}\right)_{\text {exp.,mixmode }}=\sum_{\alpha=1,2} \Delta \omega_{\alpha} .
$$

Because $\Delta \omega_{1}$ and $\Delta \omega_{2}$ can be measured separately, as was explained in section 3.1.3, we can calculate $\left(\Delta \omega_{1 / 2}\right)_{\text {mixmode }}$ and find out at what $q$ this value equals $(\Delta \omega)_{\text {exp }}$. When the mixture mode contribution at its maximum

$$
\frac{G_{1}^{2}}{K_{1}}=\frac{G_{2}^{2}}{K_{2}}
$$

Hence from a measurement of $\mathbf{q}_{\text {mix }}$ there follows the corresponding scattering angle $\theta$, which in turn gives $G_{1}$ and $G_{2}$. The ratio $K_{1} / K_{2}$ can then be calculated with the help of equation (19). The advantage of this method is that the data are obtained from noise measurements which do not contain the typical experimental errors associated with measurements of the angular distribution of scattered light.

3.1.6 Corrections in the case of light scattering experiments on flat samples. - In our set-up involving light scattered by birefringent material the scattered light is refracted as it leaves the NLC, according to Snell's law, i.e. $\sin \theta_{\mathrm{L}}=n \cdot \sin \theta . \theta$ and $\theta_{\mathrm{L}}$ are the scattering angles inside and outside the medium respectively; $n$ is the refractive index, which, in the case of birefringent material like NLC, is angular dependent, i.e. $n=n(\theta)$.
In angular dependent light scattering investigations one has to take absorption effects and multiple scattering into account. Furthermore the measured data have to be corrected, as is described below.

i) As the scattered light leaves the NLC, part of it will be reflected and the amount of light will depend on the scattering angle and the direction of polarization. The transmissivity is based on the Fresnel formulae [11] which have to be used to calculate the original scattered light intensity.

ii) Because the detector area is finite and at a constant distance from the scattering volume, one samples within a solid angle $\mathrm{d} \Omega_{\mathrm{L}}$ with fixed dimensions. The relation between the solid angle outside and inside the medium, $\mathrm{d} \Omega_{\mathrm{L}}$ and $\mathrm{d} \Omega$, respectively is given by Chatelain [12] :

$$
\mathrm{d} \Omega_{\mathrm{L}}=n^{2} \frac{\cos \theta}{\cos \theta_{\mathrm{L}}} \mathrm{d} \Omega .
$$

iii) The measured anode current is proportional to the intensity of the scattered light and hence to the differential cross section, i.e. $\mathrm{d} \sigma / \mathrm{d} \Omega_{\mathrm{L}}$, from which $\mathrm{d} \sigma / \mathrm{d} \Omega$ can be determined according to equation (20). For the cross section, measured within the solid angle $\mathrm{d} \Omega$, one can write :

$$
\mathrm{d} \sigma \sim \int_{\alpha_{\mathrm{dq}}} \int A(\mathbf{q}, 0) \mathrm{d} \mathbf{q} \simeq A(\mathbf{q}, 0) \cdot \alpha_{\mathrm{dq}},
$$

where $\alpha$ is the area in $\mathbf{q}$-space, consisting of all possible points representating all possible scattering vectors $q$. The subscript dq denotes that we have taken a finite area around $\mathbf{q}$, which has size $\mathbf{d q}$ and corresponds to $\mathrm{d} \Omega$. One can easily prove that $\alpha_{\mathrm{dp}}=\frac{1}{\lambda_{0}^{2}} n^{2} \mathrm{~d} \Omega$, hence

$$
\frac{\mathrm{d} \sigma}{\mathrm{d} \Omega} \sim \frac{1}{\lambda_{0}^{2}} n^{2} A(\mathbf{q}, 0)
$$

It should be noted that when the refractive index is angular-dependent, equation (21) has to be taken into account.

\subsection{THE DISTORTION VISCOSITY AS A FUNCTION OF} TEMPERATURE. - Experimental values for static physical quantities of NLC seem to be in reasonable agreement with theoretical values. Very few data on the dynamical physical quantities are available and comparisons with theory are rare.

3.2.1 Relations between the splay and twist viscosities and the Leslie coefficients. - The viscous properties of NLC are described by six so-called Leslie coefficients [13]. The Orsay-group [1] derived the following expressions for the splay $\left(\eta_{1}\right)$ and twist $\left(\eta_{2}\right)$ viscosities in terms of the Leslie coefficients :

$$
\eta_{1}=\gamma_{1}-\alpha_{3}^{2} / \eta_{\mathrm{b}} \text { and } \eta_{2}=\gamma_{1} \text {, }
$$


where $\gamma_{1}=\alpha_{3}-\alpha_{2}$ is the rotational viscosity and $\eta_{\mathrm{b}}=\frac{1}{2}\left(\alpha_{3}+\alpha_{4}+\alpha_{6}\right)$ is a Mięsowicz constant. Although the Leslie coefficients may be negative, the viscosities such as $\eta_{1}, \eta_{2}, \eta_{\mathrm{b}}$ and $\gamma_{1}$ must always be positive. Hence we can write :

$$
\frac{\eta_{1}}{\eta_{2}}=1-\frac{\alpha_{3}^{2}}{\eta_{\mathrm{b}} \cdot \gamma_{1}} \leqq 1 .
$$

From investigations on MBBA [14] and DIBAB [15] it is concluded that $\alpha_{3}$ is very small compared to $\eta_{\mathrm{b}}$ and $\gamma_{1}$. Therefore $\eta_{1} \approx \eta_{2}$.

From our experiments we derive the ratios $\eta_{1} / K_{1}$, $\eta_{2} / K_{2}$ and $K_{1} / K_{2}$. Hence from these data we are able to determine the ratio $\eta_{1} / \eta_{2}$ and compare this with equation (22).

3.2.2 The viscoelastic ratio as a function of temperature and ordering. - The elastic and the viscosity constants are expected to depend on the state of alignment of the rod-like molecules of the NLC [16]. The state of alignment can be given by an order parameter $S$, defined by $S \equiv \frac{3}{2}\left\langle\cos ^{2} \theta\right\rangle-\frac{1}{2}$, where $\theta$ is the angle between the elongated axis of a molecule and the director. As shown in the literature $[17,18]$ the anisotropy of the mass susceptibility, i.e. $\Delta \chi^{\mathrm{g}}$, is linear with this order parameter $S$, i.e.

$$
\Delta \chi^{\mathrm{g}} \equiv \frac{\left(\chi_{\|}-\chi_{\perp}\right)}{\rho}=c . S .
$$

Here $\chi_{\|}$and $\chi_{\perp}$ are the magnetic susceptibility constants for magnetic fields parallel and perpendicular to $\mathbf{n}_{0}$ respectively. If $\Delta \chi^{\mathbf{g}}$ is measured as a function of temperature one finds $S$ as a function of temperature [19]. The elastic as well as the viscosity constants also depend on the order parameter $S$. Several authors [19-21] have concluded from calculations and measurements that for nematics the Frank elastic constants are proportional to $S^{2}$. Hence $K_{\alpha} \approx c_{\alpha} \cdot S^{2}$ ( $\alpha=1,2$ or 3 , representing splay, twist or bend respectively), where $c_{\alpha}$ is a constant that depends only on molecular properties. Martins and Diogo [7] derived that $S$ was dependent on the rotational viscosity. Unfortunately there have been very few investigations on the dynamical quantities of NLC. By measuring $\eta_{1}$ and $\eta_{2}$ as a function of temperature and by measuring the temperature dependence of $S$, one can also determine the $S$ dependences of $\eta_{1}$ and $\eta_{2}$ and compare them with the theoretical relation.

Results reported by several authors $[6,7,14,22]$ let us consider a phenomenological relation for the twist viscosity coefficient :

$$
\eta_{2}=\gamma_{1}=S^{\beta} \cdot \mathrm{e}^{\varepsilon(S) / k T} \quad(\beta \gtrsim 0) .
$$

The exponential factor is quite a usual one for normal or isotropic liquids [23]. The energy term $\varepsilon(S)$ is the so-called activation energy for one particle. We can assume that $\varepsilon(S)$ is proportional to $S$ according to the mean field theory [24], i.e. $\varepsilon(S)=\varepsilon_{0} S$ [7]. In spite of this, several authors $[6,14]$ find that $\varepsilon(S)$ is independent of $S$, i.e. $\varepsilon(S)=\varepsilon_{0}$. They consider $\varepsilon$ as an activation energy for the rotation of a molecule around an axis perpendicular to the long axis of the molecule. In other words, if $\varepsilon=\varepsilon_{0} S$, then this activation energy is due to collective interaction effects. On the other hand, if $\varepsilon=\varepsilon_{0}$, this energy is only related to individual effects of the molecules.

4. Experimental results. - For typical plots of experimental results which we derived with the help of our experimental arrangement, we refer to papers recently published by van Eck and Zijlstra [2, 25]. Our experimental investigations have led us to conclude that absorption effects and multiple scattering need not be taken into account because they are negligible. It has been verified that the observed scattered light is due only to director-fluctuations. This was done by measuring light scattered by the same sample but at a temperature above the clearing temperature $T_{\mathrm{NI}}$. In this case the observed detector current is not due to light scattering caused by director-fluctuations but is caused by density fluctuations, reflection effects, or dark current. It turns out that in this case the measured anode current is smaller than 0.001 of the anode current caused by scattering by crystals in the nematic phase. It should be noted that we worked with crossed polarizers.

Because the dimensions of the solid angle of observation are not infinitely small one measures in fact over a certain distribution of scattering angles. We have calculated, however, that the ensuing systematic error is much smaller than the experimental errors, so it can be neglected.

Table I shows the measured data for the splay/twist viscoelastic ratios at different temperatures, obtained from noise measurements. In this table data are also given for the splay/twist elastic ratios obtained from light intensity measurements and from noise measurements in the mixture mode.

4.1 Viscoelasticity. - The viscoelastic ratios are derived using the methods outlined in section 3.1.4. The scattering angle $\theta_{\mathrm{L}}$ was varied between $5^{\circ}$ and $25^{\circ}$ so that only homodyne data were measured, which were not influenced by any heterodyne mixing. Heterodyne mixing will occur when the detected light mixes with the original laser light. Van Eck et al. [8] showed that this occurred for scattering angles of less than $3^{\circ}$. To be convinced that in a homodyne set-up the results are not influenced by any heterodyne mixing effects one can compare these results with the results from a pure heterodyne set-up. Because the bandwidth of a pure homodyne spectrum must differ from the bandwidth of the heterodyne spectrum by a factor 2 one is able to assess the purity of the homodyne spectrum. We did this by making use of the same heterodyne set-up as was used and described by van Eck and Zijlstra [2] (cf. Figs. 3 and 4 of [2]). We found 
Table I. - The measured values of the splay (1) and the twist (2) viscoelastic ratios and the splay/twist elastic ratios of $O H M B B A$.

$\begin{array}{ccc} & \eta_{1} / K_{1} & \eta_{2} / K_{2} \\ T / T_{\mathrm{NI}} & \times 10^{9} \mathrm{sm}^{-2} & \times 10^{9} \mathrm{sm}^{-2} \\ - & - & - \\ 0.9980 & 6.10 \pm 0.20 & 9.7 \pm 0.5 \\ 0.9905 & 6.41 \pm 0.12 & 11.8 \pm 0.7 \\ 0.9870 & & 13.3 \pm 1.0 \\ 0.9850 & & \\ 0.9771 & 7.04 \pm 0.15 & 14.1 \pm 0.5 \\ 0.9700 & 7.35 \pm 0.20 & 17.2 \pm 1.5 \\ 0.9620 & 8.05 \pm 0.25 & 20.0 \pm 1.0 \\ 0.9550 & 9.8 \pm 0.5 & 21.1 \pm 0.5 \\ 0.9480 & 9.6 \pm 0.3 & 23.5 \pm 0.5 \\ 0.9406 & 10.8 \pm 0.2 & 26.0 \pm 1.5 \\ 0.9324 & 12.5 \pm 0.3 & 32.3 \pm 1.0\end{array}$

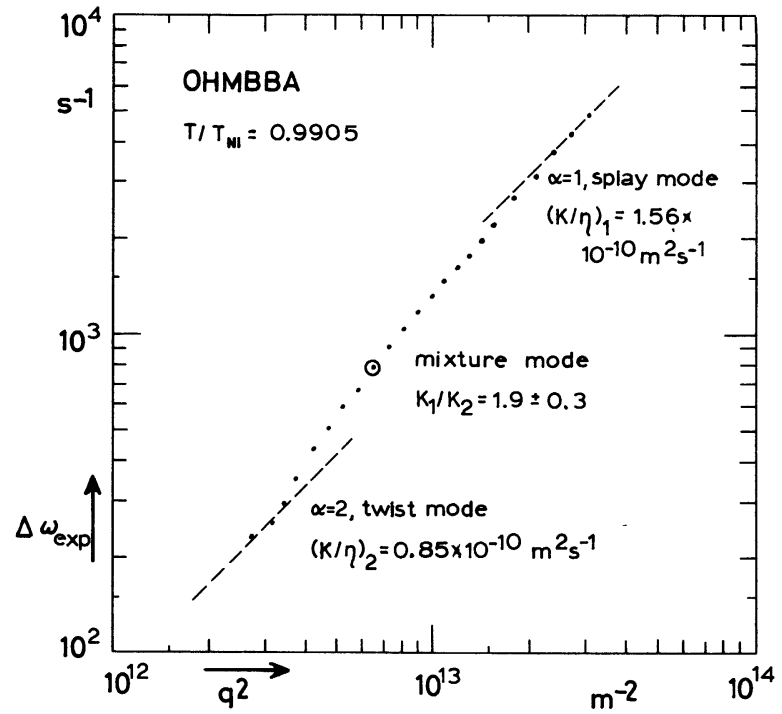

Fig. 4. - Example of a log-log plot of the measured values of. $\Delta \omega_{\exp }$ versus $q^{2}$ for $T / T_{\mathrm{NI}}=0.9905$. Because

$$
\Delta \omega_{\exp }=2 \Delta \omega_{\alpha}=2\left(K_{\alpha} / \eta_{\alpha}\right) q^{2},
$$

with $\alpha=1$ or $\alpha=2$ in the splay mode or the twist mode respectively, we expect in these modes straight lines with a slope of +1 . The ratio $K_{\alpha} / \eta_{\alpha}$ can be determined from the position of the line.

that their bandwidths at angles $>5^{\circ}$ were half the bandwidths found in the homodyne experiment, just as we had expected. It is noted that the number of coherence areas, subtended by the detector is of no importance in our case since it does not affect the measured half-bandwidths at all. A typical plot of $\Delta \omega_{\exp }$ versus $q^{2}$ is shown in figure 4 . In our configuration (cf. Fig. 2) the following relation for $q^{2}$ holds :

$$
q^{2}=\left(\frac{2 \pi}{\lambda_{0}}\right)^{2}\left\{n_{\|}^{2}+n_{\perp}^{2}-2 n_{\|} n_{\perp} \cos \theta\right\},
$$

$\begin{array}{cl}\left(K_{1} / K_{2}\right)_{\text {crosssect. }} & \left(K_{1} / K_{2}\right)_{\text {mix mode }} \\ 1.89 \pm 0.02 & 1.70 \pm 0.35 \\ 1.88 \pm 0.06 & 1.9 \pm 0.3 \\ & \\ 1.90 \pm 0.04 & \\ 2.02 \pm 0.06 & 1.9 \pm 0.3 \\ 2.06 \pm 0.08 & 1.9 \pm 0.3 \\ 2.04 \pm 0.02 & 1.7 \pm 0.3 \\ 1.94 \pm 0.04 & 1.91 \pm 0.18 \\ 1.99 \pm 0.04 & 1.8 \pm 0.3 \\ 2.09 \pm 0.06 & 1.6 \pm 0.3 \\ 2.02 \pm 0.02 & \end{array}$

where

$$
\theta=\arcsin \left(\frac{1}{n_{\perp}} \sin \theta_{\mathbf{L}}\right)
$$

and where $n_{\perp}$ and $n_{\|}$are the refractive indices of the NLC relating to the ordinary and extraordinary waves respectively ( $\perp$ and $\|$ refer to electric field polarizations perpendicular and parallel to the director respectively). In order to calculate $q^{2}$ from $\theta_{\mathrm{L}}$, we used data for $n_{\perp}$ and $n_{\|}$from Leenhouts [4]. Although the solidstate - nematic transition temperature, i.e. $T_{\mathrm{SN}}$, is $44{ }^{\circ} \mathrm{C}$ we were still able to measure somewhat below this temperature because of undercooling. Figure 5 shows the experimental results for the splay/twist

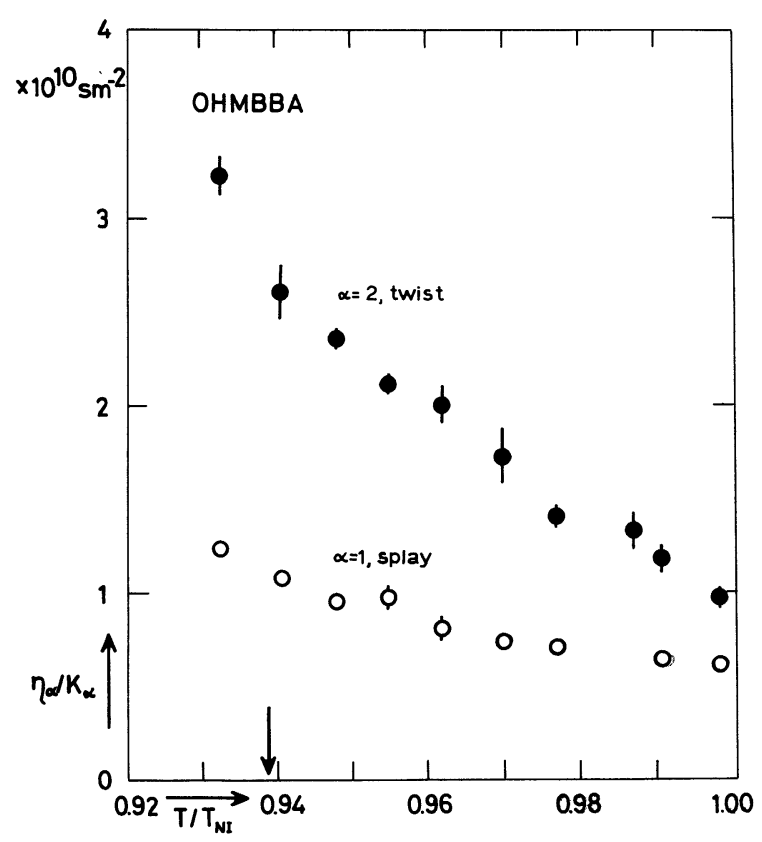

Fig. 5. - Experimental data for the viscoelastic ratios $(\eta / K)_{\alpha}$ versus $T / T_{\mathrm{NI}}$ for $\alpha=1$ (splay), circles, and $\alpha=2$ (twist), dots. 
viscoelastic ratios as a function of the reduced temperature $T / T_{\mathrm{NI}}$. The arrow indicates $T_{\mathrm{SN}} / T_{\mathrm{NI}}$.

4.2 Elasticity. - As was pointed out in section 3.1.3, we investigated the ratio between the splay and twist elastic constants $\left(K_{1}: K_{2}\right)$ at different temperatures by measuring the angular intensity distribution of the scattered light as a function of the scattering angle $\theta$, paying due attention to what we wrote in section 3.1.6.

As was proved recently by van Eck and Zijlstra [2], the time averaged anode current of the multiplier is directly proportional to the intensity of light observed by the PMT and is directly coupled with the integral of the frequency spectrum and the number of coherence areas per area of the aperture (cf. Fig. 5 of [2]). Since the solid angle subtended by the detector is independent of the scattering angle the observed anode current is proportional to the detected light intensity. Although there are alternative methods [25, 26] for deriving $K_{1}: K_{2}$ from $I(\theta)$, we have plotted the quantities $J \equiv I / Q$ versus $\left(G_{1}^{2}-G_{2}^{2}\right)$ (see Fig. 6). $I$ and $Q$

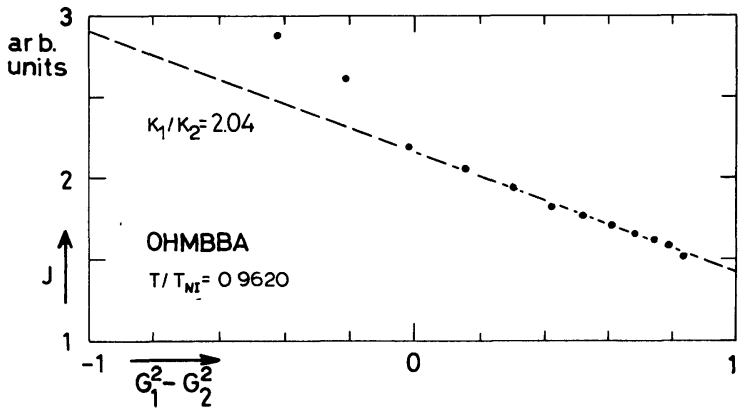

Fig. 6. - Example of a plot of $J$ versus $\left(G_{1}^{2}-G_{2}^{2}\right)$ for $T / T_{\mathrm{NI}}=0.9620$. The extrapolated values $J(+1)$ and $J(-1)$ give $J(+1) / J(-1)$ which corresponds to the elastic ratio $K_{1} / K_{2}$.

are defined in equation (15). We choose to plot $J$ because it is linear with $\left(G_{1}^{2}-G_{2}^{2}\right)$, as follows from (17) and from $G_{1}^{2}+G_{2}^{2}=1$, i.e.

$J \sim \frac{G_{2}^{2}}{K_{1}}+\frac{G_{2}^{2}}{K_{2}}=\frac{K_{1}+K_{2}}{2 K_{1} K_{2}}-\frac{K_{1}-K_{2}}{2 K_{1} K_{2}}\left(G_{1}^{2}-G_{2}^{2}\right)$.

This relation has been verified experimentally as shown in figure 6, but it has not been verified for a few points where $\left(G_{1}^{2}-G_{2}^{2}\right)$ goes to -1 . The reason for this is that at small scattering angles the scattered light is detected with unscattered light.

If one considers $J$ as a function of $G_{1}^{2}-G_{2}^{2}$ it follows from equation (25) that $K_{1} / K_{2}=J(-1) / J(+1)$. Hence it is possible to determine $K_{1}: K_{2}$ by extrapolating the curve. The range of scattering angles is limited by the dimensions of the heat stage holder.
Figure 7 shows the experimental results for the splay/ twist elastic ratio as a function of the reduced temperature $T / T_{\mathrm{NI}}$ obtained from the angular distribution of the scattered light. This figure also shows the results obtained with the mixture mode technique (cf. section 3.1.5). The dashed line is the best fit to the data from Leenhouts [19]. $T_{\mathrm{SN}} / T_{\mathrm{NI}}$ is indicated by an arrow.

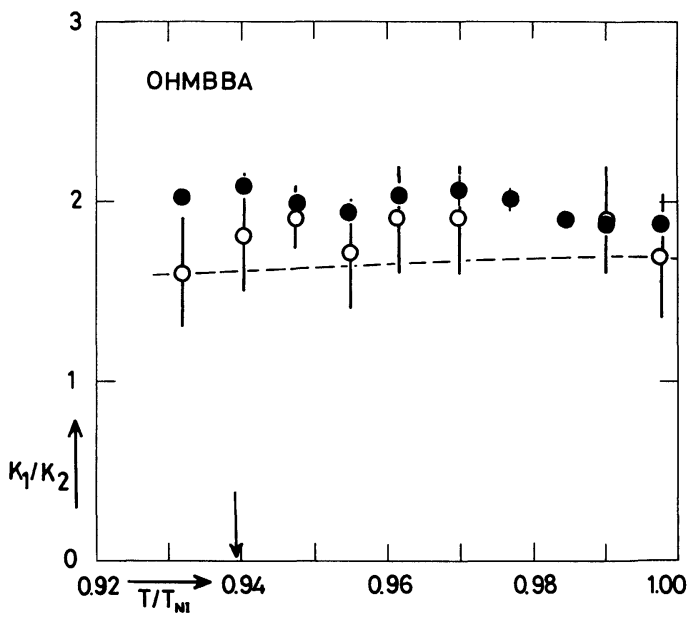

Fig. 7. - The elastic ratio $K_{1} / K_{2}$ versus the reduced temperature $T / T_{\mathrm{Nl}}$. The dots are from the intensity measurements. The circles are from the spectral density measurements. The broken curve is from Freedericksz transition data [19].

5. Discussion. - 5.1 Viscosity. - As was suggested in section 3.2 .1 , we plot $\eta_{1} / \eta_{2}$ versus the reduced temperature, as is shown in figure 8 . In order to calculate $\eta_{1} / \eta_{2}$ we can use the data for $K_{1}: K_{2}$ obtained from the angular dependency distribution of the scattered light. We can conclude that for all points except one $\eta_{1} / \eta_{2} \leqq 1$, as expected from equation (22).

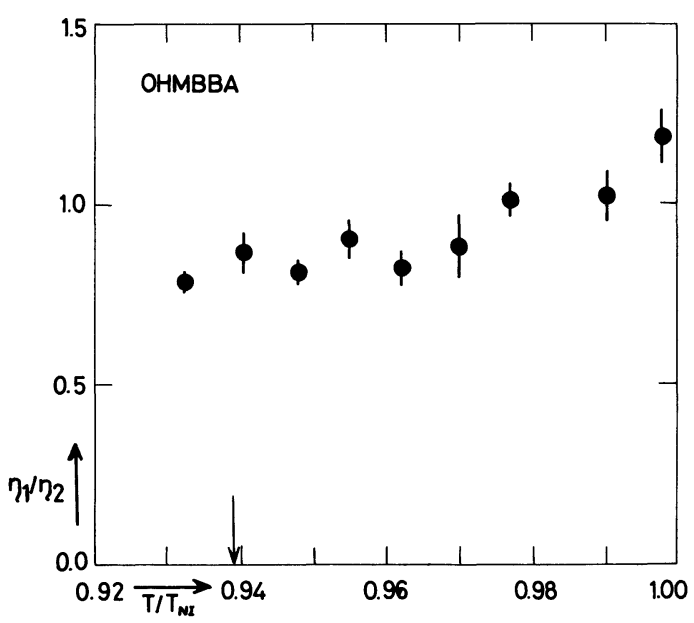

Fig. 8. $-\eta_{1} / \eta_{2} \equiv \eta_{\text {splay }} / \eta_{\text {twist }}$ versus $T / T_{\mathrm{NI}}$, from calculations of $\eta_{2} / K_{2}$ and $\eta_{1} / K_{1}$ with $\left(K_{1} / K_{2}\right)$ obtained from the angular dependency of the scattered light. 
Investigations of the order and temperature dependence of $\eta_{1}$ and $\eta_{2}$ were carried out as suggested in 3.2.2 using experimental data for $\Delta \chi^{\mathrm{g}}, K_{1}$ and $K_{2}$ from Leenhouts $[18,19]$. Because neither $\beta$ nor $\varepsilon(S)$ can be determined directly we chose values for $\beta$ and $\delta(S)$ and then tested them. We chose $\beta=0,1$ and 2 and $\varepsilon(S)=\varepsilon_{0} S$ and $\varepsilon_{0}$. By dividing the values of $\eta_{2}$ and $\eta_{1}$ by $\left(\Delta \chi^{\mathrm{g}}\right) \sim(S)^{\beta}$ we compensate the factor $S^{\beta}$. The logarithmic value of these results ought then to be of the type $\varepsilon(S) / k T$. Assuming that $\varepsilon(S) / k T \sim 1 / T$ or $\sim S / T$ we can now plot these values versus $T^{-1}$ or $\Delta \chi^{\mathrm{g}} / T$. The curve which fits best to a straight line then provides us with the most acceptable values for $\beta$ and $\varepsilon(S)$.

In figures $9 a$ to $9 f$ six plots are shown; we can conclude that the rotational or twist viscosity shown in figure $9 f$ fits best to a straight line. It follows that

$$
\eta_{2}=\gamma_{1}=c \cdot \exp \left(\varepsilon_{0} S / k T\right) .
$$

As a matter of fact, if we, like Prost [6], consider the kinematic viscosity $v_{\alpha}=\eta_{\alpha} / \rho$ instead of the dynamic viscosity $\eta_{x}$ no difference is observed, within the accuracy of the data. Since the $T$ and $S$ dependence of $\gamma_{1}$ is determined mainly by the exponential, the exponent of the pre-exponential factor cannot be determined unambiguously. One can only hope to obtain better information about the pre-factor in the range of temperatures where $S$ changes strongly with temperature. Therefore measurements of $\gamma_{1}$ will have to be done in the proximity of the nematic isotropic transition temperature.

From the slope of the curve in figure $9 f$ we calculated $\varepsilon_{0}$ in equation (26), where we took $S_{\mathrm{NI}}=0.31$ from Leenhouts [18]. We get $\varepsilon_{0}=0.22 \mathrm{eV}$, while from the mean field theory [24] it follows that $\varepsilon_{0} \approx 0.2 \mathrm{eV}$, so these results are in rather good agreement.

As was pointed out in section 3.2.1 the splay viscosity should be written as a combination of viscosity terms. On the other hand, there is no theoretical analysis available from which we can predict how $\eta_{1}$ will behave as a function of temperature or of order. Figure $9 f$ shows, however, that this dependence is the same as that found for $\eta_{2}$ but not at temperatures close to the nematic isotropic transition temperature.

5.2 Elasticity. - Figure 7 shows that the data for the splay/twist elastic ratio obtained from the angular distribution of the scattered light and those obtained from the mixture mode agree within the accuracy of the measurements. Data obtained from Freedericksz transitions [19], however, are about $10 \%-20 \%$ lower than our values. This systematic difference was also observed for other nematics such as $\mathrm{N}_{4}$ and DIBAB $[25,27]$. At present no satisfactory reason for this difference has been found. One argument could be that in the case of the Freedericksz experiment the condition of strong anchoring was not fulfilled, although investigations done with a variety of sample thicknesses [19] demonstrate that this condition
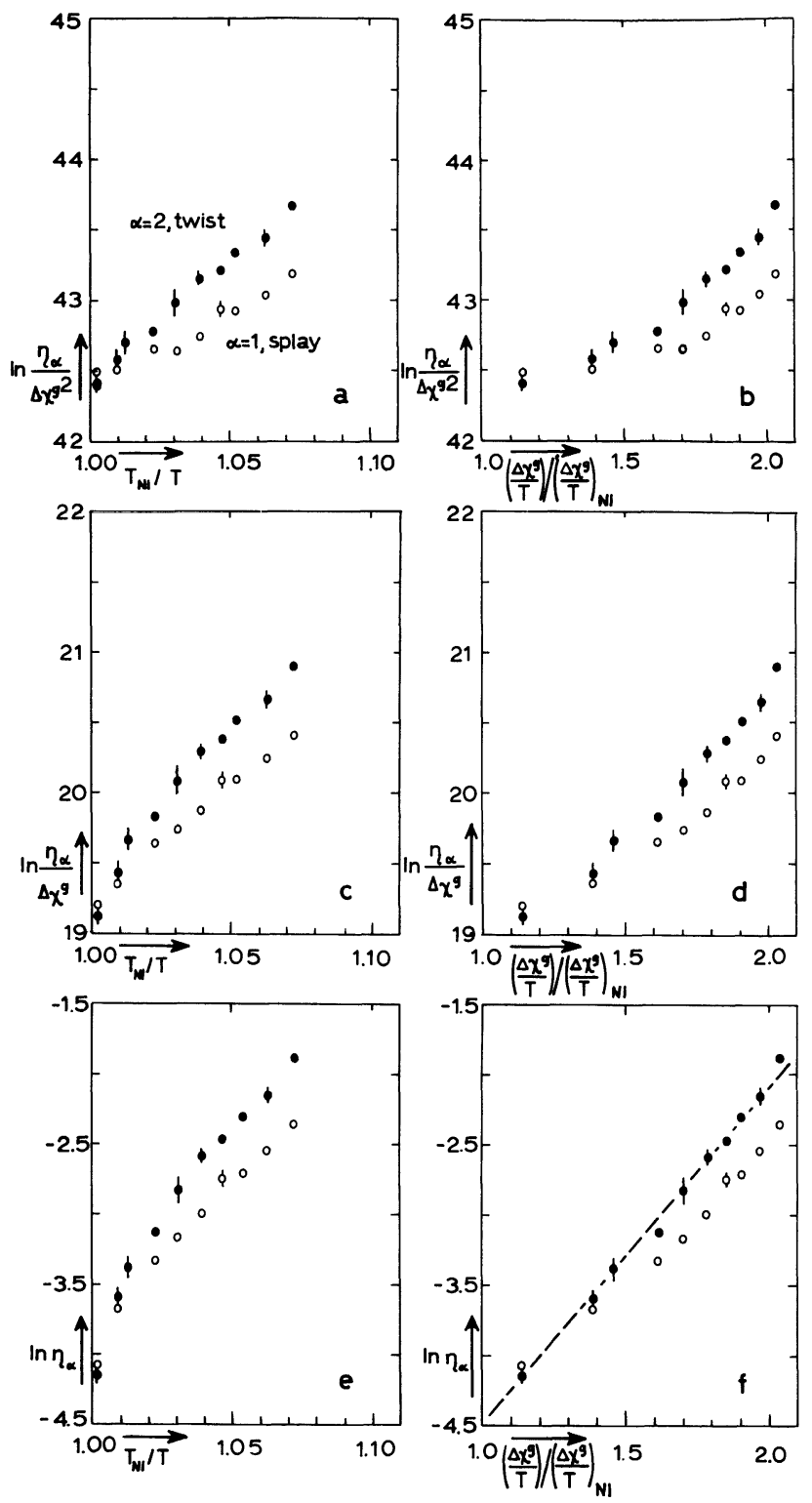

Fig. 9. - The natural logarithm of combinations of $\eta_{\alpha}$ and $\Delta \chi^{\mathrm{g}}$ with $\alpha=1$ (splay), circles, and $\alpha=2$ (twist), dots, plotted versus $T_{\mathrm{NI}} / T \sim T^{-1}$ and versus

$$
\left(\Delta \chi^{\mathrm{g}} / T\right) /\left(\Delta \chi^{\mathrm{g}} / T\right)_{\mathrm{NI}} \sim S / T
$$

in order to determine the order parameter dependency of the twist viscosity. Obviously $\eta_{\text {twist }}$ versus $\left(\Delta \chi^{\mathrm{g}} / T\right) /\left(\Delta \chi^{\mathrm{g}} / T\right)_{\mathrm{NI}}$ fits best a linear function, so $\eta_{\text {twist }}\left(=\gamma_{1}\right)=c \cdot \exp (\varepsilon S / k T)$.

was fulfilled. Another criticism is that the volume dependence of the Freedericksz transition is neglected in the theory because its derivation is based on the Helmholtz free energy for distortion, which implies constant volume. This assumption is doubtful, however, because the distortion of the nematic by the magnetic field will certainly change the volume. In practice one always works at constant pressure, so the Gibb's free energy should be used.

Acknowledgments. - We wish to thank Dr. W. H. de Jeu and Dr. F. Leenhouts of the University 
of Groningen for kindly supplying OHMBBA and for valuable and stimulating discussions. We are also grateful to Sheila McNab for making linguistic improvements and to Greetje Hollander for typing the manuscript.
This work was performed as part of the research programme of the "Stichting voor Fundamenteel Onderzoek der Materie » (FOM) with financial support from the « Nederlandse Organisatie voor Zuiver Wetenschappelijk Onderzoek » (ZWO).

\section{References}

[1] Groupe D'Etude des Cristaux Liquides d'Orsay, J. Chem. Phys. 51 (1969) 816.

[2] VAN ECK, D. C. and ZiJlstra, R. J. J., J. Physique 41 (1980) 351

[3] Schaetzing, R. and Litster, J. D., in Advances in Liquid Crystals, Vol. IV, edited by Brown, G. H. (Acad. Press, New York) 1979, p. 147.

[4] Leenhouts, F., Private communication; see also : Leenhouts, F., J. Physique-Lett. 39 (1978) L-249.

[5] Chatelain, P., Acta Crystallogr. 4 (1951) 453.

[6] Prost, J., Sigaud, G. and Regaya, R., J. PhysiqueLett. 37 (1976) L-341.

[7] Martins, A. F. and Diogo, A. C., Portugal Phys. 9 (1975) 129 ; see also :

Diogo, A. C. and Martins, A. F., Mol. Cryst. Liq. Cryst. 66 (1981) 133.

[8] Van Eck, D. C. and Westera, W., Mol. Cryst. Liq. Cryst. 38 (1977) 319.

[9] De Gennes, P.-G., C.R. Hebd. Séan. Acad. Sci. B 266 (1968) 15.

[10] Berne, B. J. and Pecora, R., Dynamic Light Scattering (John Wiley and Sons, New York) 1976, p. 27.

[11] Born, M. and Wolf, E., Principles of Optics (Pergamon Press, Oxford) 1970, p. 41.

[12] Chatelain, P., Acta Crystallogr. 1 (1948) 315.

[13] Leslie, F. M., Quart. J. Mech. Appl. Math. 19 (1966) 357.

[14] De Jeu, W. H., Phys. Lett. 69A (1978) 122.

[15] Van Eck, D. C. and Perdeck, M., Mol. Cryst. Liq. Cryst. Lett. 49 (1978) 39.
[16] De Jeu, W. H., Physical Properties of Liquid Crystalline Materials (Gordon and Breach, New York) 1980.

[17] Alben, R., McColn, J. R. and Shin, C. S., Solid State Commun. 11 (1972) 1081.

[18] Leenhouts, F., De Jeu, W. H. and Dekker, A. J., J. Physique 40 (1979) 989.

[19] Leenhouts, F. and Dekker, A. J., J. Chem. Phys. 74 (1981) 1956 ; see also :

LeENhouts, F., Roebers, H. J., DekKer, A. J. and Jonker, J. J., J. Physique Colloq. 40 (1979) C3-291.

[20] Nehring, J. and Saupe, A., J. Chem. Phys. 56 (1972) 5527.

[21] Priest, R., Mol. Cryst. Liq. Cryst. 17 (1972) 129 and Phys. Rev. A 7 (1973) 720.

[22] SChadT, M. and MÜlleR, F., IEEE Trans. Electron. Devices ED-25 (1978) 1125.

[23] Frenkel, J., Kinetic Theory in Liquids (Dover Publ. Inc., New York) 1955.

[24] MaIER, W. and SAUPE, A., Z. Naturforsch. A 13 (1958) 564, A 14 (1959) 882, A 15 (1960) 287.

[25] Van Eck, D. C. and ZiJlstra, R. J. J., in Noise in Physical Systems, edited by Wolf, D. (Springer Verlag, Berlin) 1978 , p. 270 ; see also :

VAN ECK, D. C., Thesis (Utrecht) 1979.

[26] Usui, H., Takezoe, H., Fukuda, A. and Kuze, E., Japan. J. Appl. Phys. 18 (1979) 1599.

[27] De Jeu, W. H. and Claassen, W. A. P., J. Chem. Phys. 67 (1977) 3705. 